\title{
HOLMES, an experiment for a direct measurement of neutrino mass
}

\author{
Andrei Puiu $^{* 3,4}$, B.Alpert ${ }^{2}$, M. Balata ${ }^{1}$, D. Bennett ${ }^{2}$, M. Biasotti ${ }^{5}$, C. Boragno ${ }^{5}$, C. \\ Brofferio $^{3,4}$, V. Ceriale ${ }^{5}$, D. Corsini ${ }^{5}$, P. K. Day ${ }^{10}$, M. De Gerone ${ }^{5}$, R. Dressler ${ }^{6}$, M. \\ Faverzani $^{3,4}$, E. Ferri ${ }^{3,4}$, J. Fowler ${ }^{2}$, F. Gatti ${ }^{5}$, A. Giachero ${ }^{3,4}$, J. Hays-Wehle ${ }^{2}$, S. \\ Heinitz $^{6}$, G. Hilton ${ }^{2}$, U. Köster ${ }^{7}$, M Lusignoli ${ }^{8}$, M. Maino ${ }^{3,4}$, J. Mates ${ }^{2}$, S. Nisi ${ }^{1}$, R. \\ Nizzolo $^{3,4}$, A. Nucciotti ${ }^{3,4}$, G. Pessina ${ }^{3}$, G. Pizzigoni ${ }^{5}$, S. Ragazzi ${ }^{3,4}$, C. Reintsema ${ }^{2}$, \\ M. Ribeiro Gomez ${ }^{9}$, D. Schmidt ${ }^{2}$, D. Schumann ${ }^{2}$, M. Sisti ${ }^{3,4}$, D. Swetz ${ }^{2}$, F. \\ Terranova $^{3,4}$, J. Ullom ${ }^{2}$ \\ ${ }^{1}$ Laboratori Nazionali del Gran Sasso (LNGS), INFN, Assergi (AQ), Italy \\ ${ }^{2}$ National Institute of Standards and Technology (NIST), Boulder, Colorado, USA \\ ${ }^{3}$ Istituto Nazionale di Fisica Nucleare (INFN) sezione di Milano Bicocca, Milano, Italy \\ ${ }^{4}$ Università degli Studi di Milano Bicocca, Milano, Italy \\ ${ }^{5}$ Istituto Nazionale di Fisica Nucleare (INFN), Sezione di Genova and Dipartimento di Fisica, \\ Università di Genova, Genova, Italy \\ ${ }^{6}$ Paul Scherrer Institut (PSI), Villigen, Switzerland \\ ${ }^{7}$ Institut Laue-Langevin (ILL), Grenoble, France \\ ${ }^{8}$ Istituto Nazionale di Fisica Nucleare (INFN), Sezione di Roma 1, Roma, Italy \\ ${ }^{9}$ Multidisciplinary Centre for Astrophysics (CENTRA-IST), University of Lisbon, Lisbon, \\ Portugal \\ ${ }^{10}$ Jet Propulsion Laboratory, California Institute of Technology, Pasadena, California, USA \\ E-mail: andrei.puiu@mib.infn.it
}

\begin{abstract}
The European Research Council has recently founded HOLMES, an experiment for a direct neutrino mass measurement with a sensitivity as low as $0.4 \mathrm{eV}$. HOLMES will perform a calorimetric measurement of the the energy released in the Electron Capture (EC) spectrum of ${ }^{163} \mathrm{Ho}$. The neutrino mass is assessed from the end point of the spectrum; the sensitivity on the mass is strongly dependent on the exact value of the end point, still not precisely known, and on background due to pile up in the region of interest. In the following lines we present an overview of the whole project and its major challenges.
\end{abstract}

XVI International Workshop on Neutrino Telescopes,

2-6 March 2015

Palazzo Franchetti - Istituto Veneto, Venice, Italy

\footnotetext{
*Speaker.
} 


\section{HOLMES}

Measuring the neutrino mass is one of the most compelling challenges of modern physics. Experiments studying oscillation phenomena have clearly shown that neutrinos have non vanishing mass and at least three neutrinos with different masses exist; yet the absolute values of these masses re main unknown. There are several methods from which neutrino mass can be assessed: cosmological observations, neutrinoless double beta decay searches and beta or electron capture spectrum end-point studies. The latter is currently the one and only which can provide a model independent measurement of the absolute scale of neutrino mass.

Within this framework the European Research Council has recently funded HOLMES, a new experiment to directly measure the neutrino mass. HOLMES will perform a calorimetric measurement of the energy released in the electron capture decay of ${ }^{163} \mathrm{Ho}$.

This measurement was originally proposed in 1982 by A. De Rujula and M. Lusignoli [2], but only in the last decade the technological progress in detector design allowed to design a sensitive experiment.

In a calorimetric measurement the energy released in the decay process is entirely contained into the detector [1], except for the fraction taken away by the neutrino. The typical spectral shape is shown in Figure 1; the region of interest for the neutrino mass measurement is the upper tail of the spectrum. The calorimetric approach eliminates both the problematic issues connected to the use of an external sources and the systematic uncertainties arising from decays on excited final states.

The most suitable detectors for this type of measurement are low temperature thermal detectors, where all the energy released into an absorber is converted into a temperature increase that can be measured by a sensitive thermometer directly coupled with the absorber. HOLMES will deploy a large array of low temperature microcalorimeters with implanted ${ }^{163}$ Ho nuclei. The resulting mass sensitivity will be as low as $0.4 \mathrm{eV}$, depending on the Q-value of the transition which lacks a precise measurement, ranging from $2.3 \mathrm{keV}$ to $2.8 \mathrm{keV}$, with a recommended value of $2.555 \pm 0.016$ $\mathrm{keV}[3]$.

HOLMES will be an important step forward in the direct neutrino mass measurement with a calorimetric approach as an alternative to spectrometry. It will also establish the potential of this approach to extend the sensitivity down to $0.1 \mathrm{eV}$ and lower.

In order to reach the sensitivity of $0.4 \mathrm{eV}$ it is necessary to collect statistics higher than $10^{13}$ decays. To fulfil this task the best experimental configuration has been defined after Monte Carlo simulations [5]: in its optimal configuration HOLMES will collect about $3 \cdot 10^{13}$ decays with an instrumental energy resolution of about $1 \mathrm{eV}$ FWHM and a time resolution of about $1 \mu \mathrm{s}$. For a total measuring time of 3 years, this requires a total ${ }^{163} \mathrm{Ho}$ activity of about $3 \cdot 10^{5} \mathrm{~Bq}$. These ${ }^{163} \mathrm{Ho}$ nuclei will be implanted in 1000 detectors.

\section{Detector Production and isotope embedding}

HOLMES, in its final configuration will deploy a 1000 pixel array of low temperature microcalorimeters: each calorimeter consists of an absorber, where the ${ }^{163}$ Ho nuclei will be implanted, coupled to a Transition Edge Sensor thermometer. In order to perform the calorimetric 
measurement of the EC spectrum, about $6.5 \cdot 10^{13}{ }^{163} \mathrm{Ho}$ must be embedded in the absorber of each calorimeter. To avoid spectral distortion at the end point due to chemical shifts, only metallic Holmium must be implanted in the absorber. The embedding system, which will also include a magnetic/electrostatic mass analyser for separating ${ }^{163} \mathrm{Ho}$ from any other disturbing nuclide, will be set-up at the GENOVA INFN laboratory.

The TES for HOLMES will Mo/Cu TES on $\mathrm{SiN}_{x}$ membrane with bismuth absorbers. The TES microcalorimeters will be fabricated in a two step process. The first steps will be carried out at the National Institute for Standard and Technology (NIST, Boulder, Co, USA) where the devices will be fabricated up to the deposition of the first $1.5 \mu \mathrm{m}$ bismuth layer. The detectors will be then finalized at the Genova INFN laboratory where a layer $10 \mu \mathrm{m}$ layer of $\mathrm{Au}:{ }^{163} \mathrm{Ho}$ will be deposed; then the bismuth absorber will be completed with a deposition of a second $1.5 \mu \mathrm{m}$ bismuth layer to fully encapsulate the 163 Ho source. The last step will be a Deep Reactive Ion Etching of the back of the silicon wafer to release the membranes with the TES microcalorimeters.

The TES array is presently being designed with the aim of achieving an energy resolution $\Delta E_{F W H M}$ of about $1 \mathrm{eV}$ at the spectrum endpoint and a time resolution $\tau_{R}$ as close as possible to 1 $\mu s$ in order be able to efficiently discard pile up events. This requires an optimal thermal design of all detector components. To minimize the stray electrical inductance $\mathrm{L}$ which limits the pulse rise time, the TES will be arranged in $2 \times 32$ sub-arrays. This arrangement allows also to maximize the geometrical filling factor and therefore the ${ }^{163} \mathrm{Ho}$ implantation efficiency.

\section{Read-out and data acquisition}

During the data acquisition period, HOLMES will be operating an array of 1000 detectors at a working temperature of $\sim 90 \mathrm{mK}$ : to read out so many TES inside the dilution refrigerator where the experiment will be mounted, is no trivial matter. The signal from each detector will be read out using a multiplexing system, whose performances are crucial for preserving the performance of the individual detector, especially in terms of signal bandwidth so that the time resolution $\tau_{R}$ will not be affected. At the moment, the most appealing read out technique applicable to large arrays of Transition Edge Sensors is rf-SQUID multiplexing [6]. It is based on the use of rf-SQUIDs as input devices with flux ramp modulation for linearisation purposes; the rf-SQUID is then coupled

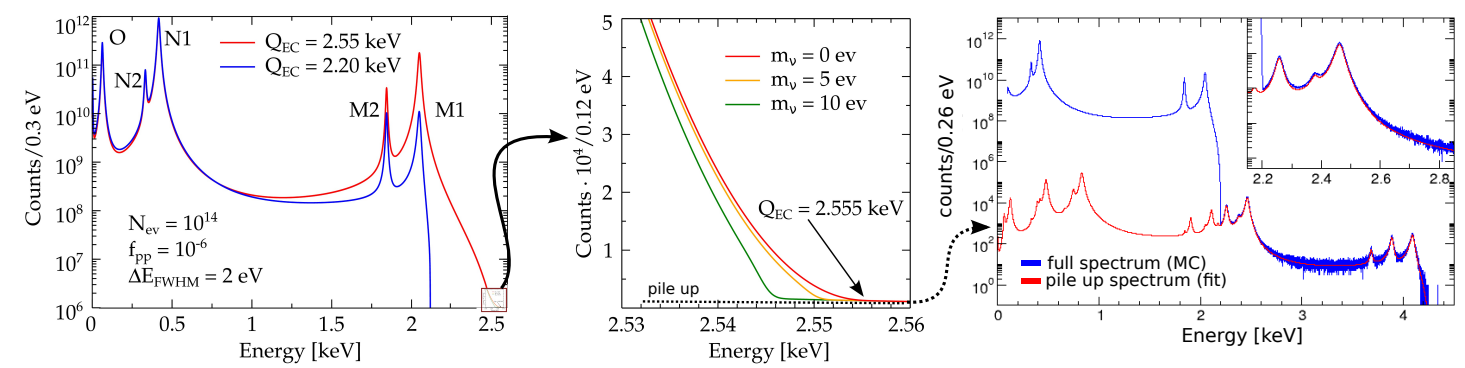

Figure 1: ${ }^{163}$ Ho total absorption spectrum calculated with energy resolution $\triangle E_{F W H M}$ of $1 \mathrm{eV}$ and pile up fraction $f_{p p}=10^{-6}$ (left). Blow up of the spectrum close to the endpoint (center). Monte Carlo simulated full spectrum with $\mathrm{Q}=2200 \mathrm{eV}$ and for $N_{e v}=10^{14}, f_{p p}=10^{-6}$ and $\Delta E_{F W H M}=1 \mathrm{eV}$ (dark line) and fitted pile up spectrum (red line). 


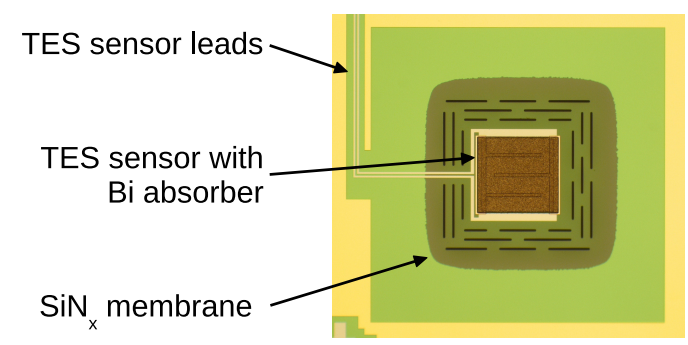

Figure 2: One TES with bismuth absorber fabricated by NIST.
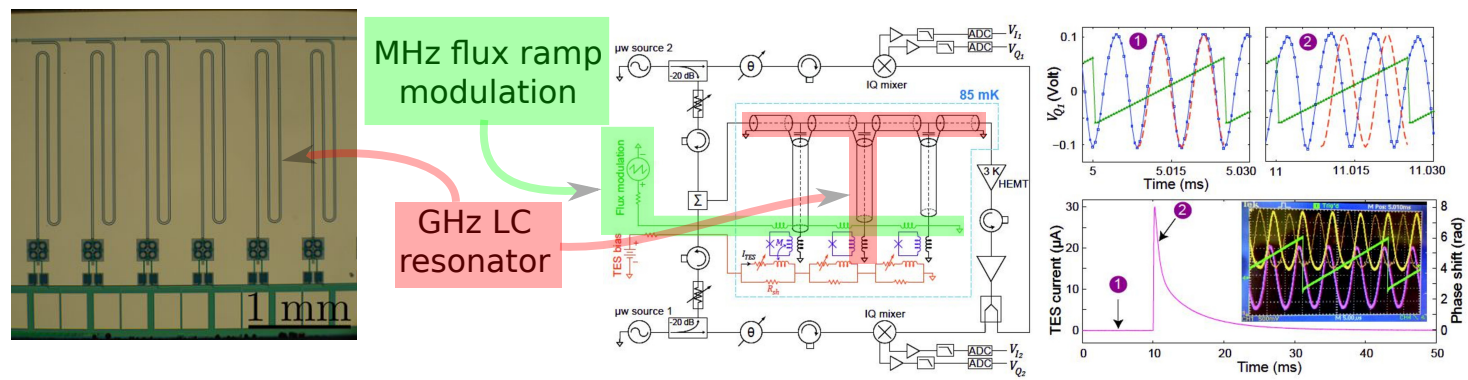

Figure 3: Circuit scheme for a two channel microwave multiplexed readout of a TES array [6].

to a super-conductive LC resonator in the $\mathrm{GHz}$ range, and the modulated signal is finally read out using the homodyne technique. A scheme of a two channel multiplexing is shown in Figure 3. Currently we are testing the performances of the multiplexing circuit and in the very near future we are planning to perform the first calorimetric measurements of the spectrum of ${ }^{163}$ Ho with high resolution in order to determine the exact value of the Q-value of the electron capture decay, which is a crucial parameter for establishing the potential sensitivity of HOLMES.

\section{Acknowledgments}

The HOLMES experiment is funded by the European Research Council under the European Union's Seventh Framework Programme (FP7/2007-2013)/ERC Grant Agreement no. 340321.

\section{References}

[1] C. Enss (Ed.), Cryogenic Particle Detection, Springer, Berlin, Heidelberg, 2005.

[2] A. De R'ujula and M. Lusignoli, Phys. Lett. B 118, (1982) 429.

[3] C. W. Reich and B. Singh, Nuclear Data Sheets 111, (2010) 1211.

[4] A. De R'ujula, arXiv:1305.4857

[5] A. Nucciotti, Eur. Phys. J. C 74, (2014) 11, arXiv:1405.5060.

[6] O. Noroozian et al., Applied Phys. Lett. 103, (2013) 202602. 